Journal of The Electrochemical Society, 2006, Volume 153, Issue 2, Pages B42-B51.

Print ISSN: 0013-4651

Online ISSN: 1945-7111

DOI: $10.1149 / 1.2142288$

http://scitation.aip.org/JES

http://scitation.aip.org/journals/doc/JESOAN-ft/vol_153/iss_2/B42_1.html

(C) The Electrochemical Society, Inc. 2006. All rights reserved. Except as provided under U.S. copyright law, this work may not be reproduced, resold, distributed, or modified without the express permission of The Electrochemical Society (ECS). The archival version of this work was published in Journal of The Electrochemical Society, 2006, Volume 153, Issue 2, Pages B42-B51.

\title{
Transition from Intergranular Corrosion to Intergranular Stress Corrosion Cracking in AA2024-T3
}

\author{
Xiaodong Liu, and G. S. Frankel \\ Fontana Corrosion Center, Department of Materials Science and Engineering
}

\section{B. Zoofan, and S. I. Rokhlin}

Department of Industrial, Welding, and Systems Engineering, The Ohio State University, Columbus, Ohio 43210, USA

The transition of intergranular corrosion (IGC) to intergranular stress corrosion cracking (IGSCC) in AA2024-T3 was studied using microfocal X-ray radiography, scanning electron microscopy (SEM), and electrochemistry. A constant potential was applied in $1 \mathrm{M} \mathrm{NaCl}$ solution, and a fixed elastic tensile displacement was applied in the transverse direction using a modified ASTM G49 jig. In this orientation, IGC grows primarily along the elongated grain boundaries in the longitudinal and transverse directions, parallel to the applied stress. SEM and X-ray radiography provided insightful images of the transition to IGSCC. Linking of the individual IGC sites occurred at the edges of the elongated grains, resulting in transition of the IGC sites into IGSCC that was nominally normal to the applied stress. This coalescence process was discontinuous in nature, as evidenced by arrest marks on the fracture surface and oscillations in the measured current.

High-strength aluminum alloys are susceptible to intergranular corrosion (IGC) and stress corrosion cracking (SCC). SCC in aluminum alloys is almost exclusively intergranular. ${ }^{1-3}$ The relationship between IGC and intergranular stress corrosion cracking (IGSCC) has been discussed for decades. ${ }^{4-7}$ The combination of stress and electrochemical reactions can alter the local environment at the IGC tips, which can accelerate the growth rate. It has been suggested that an intergranular (IG) crack subjected to a normal tensile stress can develop into IGSCC. However, the influence of tensile stress parallel to a crack tip on the subsequent growth is unclear, as are the details of how IGC transitions into IGSCC. In order to understand this transition, IGC and SCC need to be monitored in situ. Zhao et al. used X-ray radiography, a nondestructive evaluation (NDE) technique, to study IGC and exfoliation corrosion in AA2024 and AA7178. ${ }^{9}$ We have described the use of X-ray radiography to image in situ the initiation and growth of multiple IGC sites in AA2024 growing in the longitudinal direction with an applied normal tensile stress in the short transverse direction. ${ }^{10,11}$ A competition between the IG cracks was observed. The deepest crack at the beginning of the experiment was found to slow and stop growing, and was then surpassed by another crack that eventually penetrated through the sample. The results of the radiography technique were found to be in agreement with data generated using the stressed foil penetration method. ${ }^{12}$ Although they are quite different, both approaches can accurately determine the rate of the fastest growing crack. In the current study, microfocal X-ray radiography was put to further use in the study of IGC and SCC in AA2024-T3. In 
particular, the effect of a stress applied in the transverse direction of a rolled plate was studied.

\section{Experimental}

A wrought AA2024-T3 plate (Cu 4.5\%, Mg 1.45\%, Mn 0.57\%, Si 0.11\%, Fe 0.25\%, Zn $0.09 \%$, Ti $0.02 \%$, Cr $0.01 \%$, and other elements total $0.05 \%$ max), with $19-\mathrm{mm}$ thickness was purchased from Metalmen Sales, Inc. The microstructure of the plate is shown in Fig. 1. Sheet samples were sliced from the plates in specific orientations relative to the rolling direction using electrical discharge machining (EDM). Figure 2 schematically shows the nomenclature used for sliced samples. The three perpendicular directions and their normal faces are referred to as $\mathrm{S}$ (short transverse), $\mathrm{T}$ (transverse), and L (longitudinal or rolling direction). Unstressed samples are referred to by the direction normal to the exposed surface, and stressed samples are referred to by two letters: the direction normal to the exposed surface and the direction of the applied stress. For example, the sample designated $\mathrm{L}-\mathrm{T}$ was stressed in the $\mathrm{T}$ or transverse direction and the $\mathrm{L}$ or longitudinal section was exposed to solution, allowing cracking in the $\mathrm{L}$ direction.

Samples were stressed along their length in the elastic region by a modified ASTM G49 fixed-displacement jig. ${ }^{12}$ The jig applied an initial strain in the range of $0.28-0.5 \%$, corresponding to a stress of 180-280 MPa. The tested material had a yield strain of $0.7 \%$ and a yield stress of $350 \mathrm{MPa}$, so the test conditions were at $40-70 \%$ of the yield strain and $50-80 \%$ of the yield stress. The residual stress in the material was not assessed, but any residual stress present would be added to the applied stress. The sheets had dimensions of $79 \times 5 \mathrm{~mm}$ and were reduced in thickness to $1.05 \mathrm{~mm}$ by grinding and polishing.

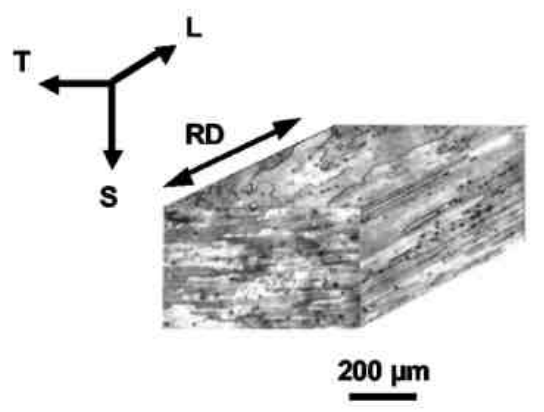

Figure 1. Microstructure and grain dimensions of $19 \mathrm{~mm}$ thickness wrought plate for AA2024-T3. RD denotes rolling direction.

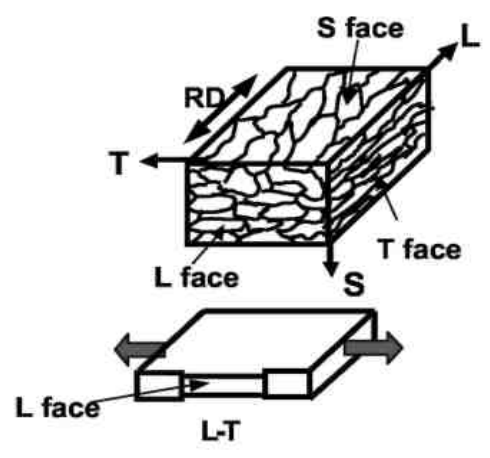

Figure 2. Notation and configuration of tested sheet sample. RD denotes the rolling direction in the L direction. L face was exposed for L-T samples. 
The narrow edge $(79 \times 1.05 \mathrm{~mm})$ of the sample was exposed to the solution so that cracks propagated along the width. A small electrochemical cell was sealed on the top of samples, allowing potentiostatic polarization of the sheet edges. The electrochemical cell has been described in detail elsewhere. Briefly, the cell was machined from a rectangular piece of Teflon with nominal dimensions of $15 \times 15 \times 23 \mathrm{~mm}$. A $1.05 \times 18.5 \mathrm{~mm}$ slot was machined in one side of the cell, and two lips that were $0.5 \times 1.5 \times 23 \mathrm{~mm}$ in dimension extended from the edges of the slot as guides for the sample. The thin edge of the sample was slipped between the lips and exposed to the inside of the cell through the slot. The cell had connections for a saturated calomel reference electrode (SCE) and a counter electrode. A $1.0 \mathrm{M} \mathrm{NaCl}$ solution was continuously pumped through the cell from a reservoir. A strip sample was inserted in the stressing jig cell, the desired displacement was applied, and then the cell was mounted on the edge of the sample. Finally, the cell was sealed along the lips with Microstop lacquer. The exposed area was about $0.2 \mathrm{~cm}^{2}(1.05 \times 18.5 \mathrm{~mm})$. The sample was potentiostatically polarized at $-580 \mathrm{mV}$ SCE to promote IGC of the AA2024-T3 and the current was recorded during the experiment. ${ }^{11,13}$ Potentiodynamic polarization was performed on AA2024-T3 in deaerated $1 \mathrm{M}$ $\mathrm{NaCl}$ at $0.1 \mathrm{mV} / \mathrm{s}$.

The details of the microfocal X-ray radiographic imaging technique and the electrochemical measurements were described previously. X-ray absorption through the sample thickness results in a negative, gray-scale, radiographic image in which higher density regions such as intermetallic particles are bright and lower density regions associated with intergranular attack or corrosion products are dark.

\section{Results}

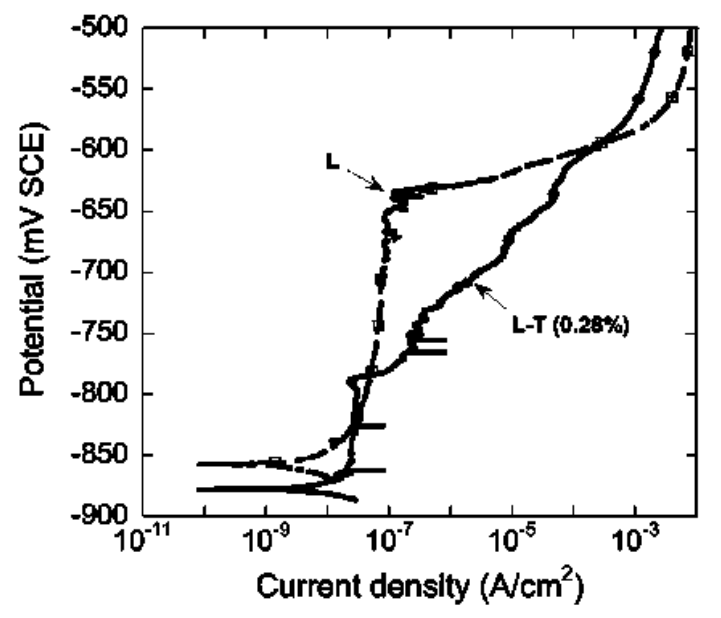

Figure 3. Potentiodynamic polarization curves for L and L-T (with $0.28 \%$ initial strain) in $1.0 \mathrm{M}$ deaerated $\mathrm{NaCl}$.

Potentiodynamic polarization of $L$ and $L-T$ samples. - Potentiodynamic polarization curves in deaerated $1 \mathrm{M} \mathrm{NaCl}$ for unstressed L surfaces and L-T samples stressed in tension to an initial strain of $0.28 \%$ are given in Fig. 3. Three replicate measurements were made for each condition and representative curves are presented. The unstressed sample exhibited one breakdown potential at an average value of $-670 \mathrm{mV}$ SCE. The L-T sample stressed to only $0.28 \%$ strain exhibited a sharp increase in current at much lower potentials; the average 
breakdown potential was only $-780 \mathrm{mV}$ SCE. However, with increasing potential the current increased at a much lower rate for the stressed sample. Metastable pitting current transients are evident in the passive range for the L-T sample. Therefore, the elastic stress seems to enhance the stability of the initial breakdown events. Similar findings were reported for stressed S-T and S-L samples compared to unstressed S samples. ${ }^{12,13}$

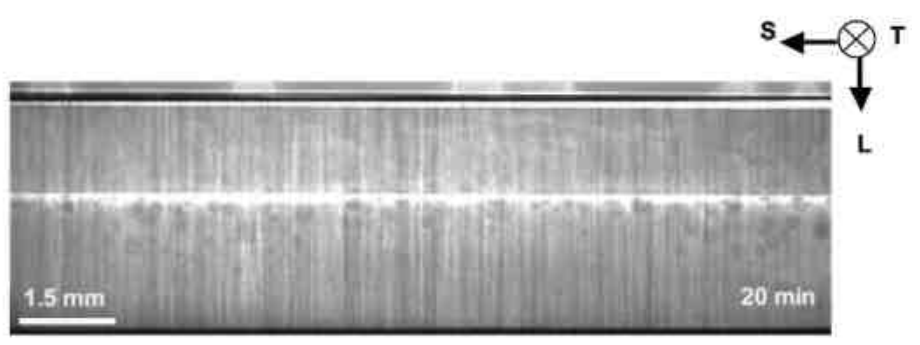

(a)

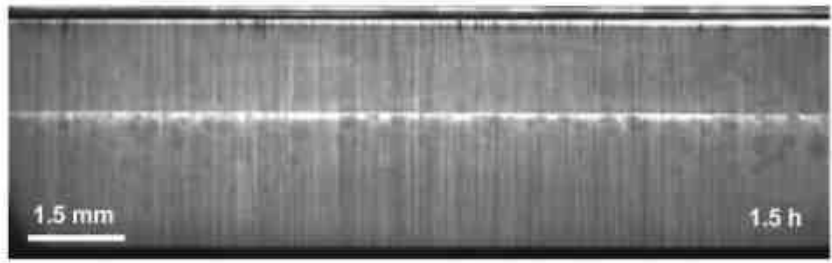

(b)

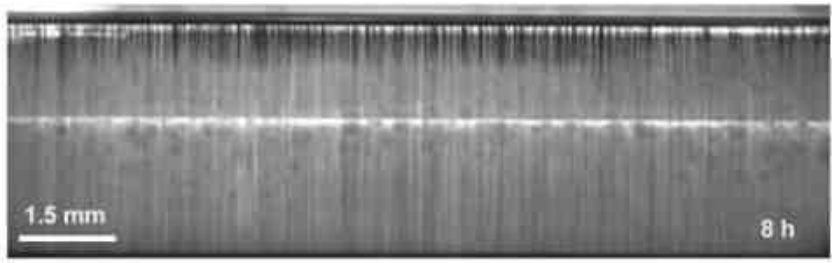

(c)

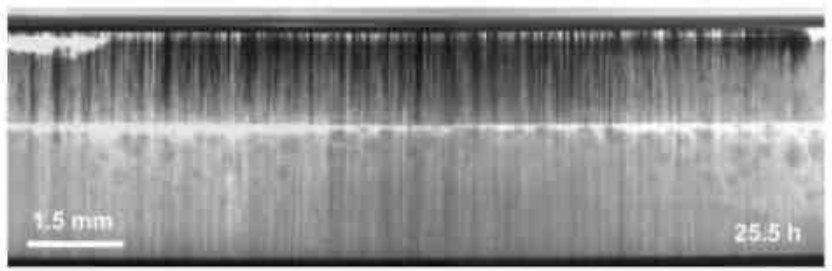

(d)

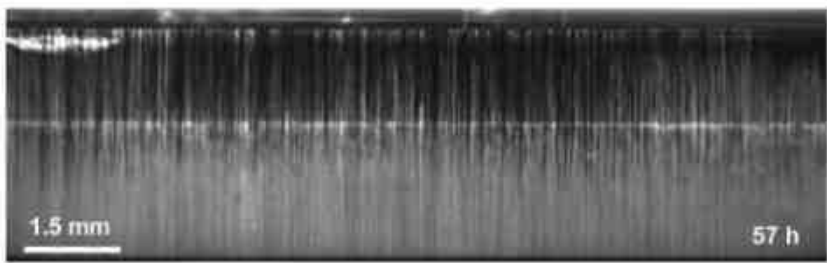

(e)

Figure 4. X-ray microfocal radiography images of IGC in unstressed L sample at $-580 \mathrm{mV}$ SCE in $1.0 \mathrm{M} \mathrm{NaCl}$ after (a) $20 \mathrm{~min}$, (b) $1.5 \mathrm{~h}$, (c) $8 \mathrm{~h}$, (d) $25.5 \mathrm{~h}$, and (e) $57 \mathrm{~h}$. 
In situ radiography of unstressed $L$ and $T$ samples. - Figure 4 shows a time sequence of radiographic images for an unstressed $\mathrm{L}$ sample during polarization in $1 \mathrm{M} \mathrm{NaCl}$ at $-580 \mathrm{mV}$ SCE. The top edge of each image was the exposed $L$ surface of the sample. The horizontal white line $1.5 \mathrm{~mm}$ below the surface is from the lacquer used to seal the end of the cell lips, ${ }^{10}$ and the vertical white streaks are the result of intermetallic particles lined up in the $\mathrm{L}$ direction. With time, dark streaks associated with IGC grew from the top exposed surface downward in the L direction. After 20 min (Fig. 4a only a few IGC sites are evident. During the time period from 1.5 to $8 \mathrm{~h}$ (Fig. 4b and c), more IGC sites initiated and their depth increased. Figure 4d shows a high density of straight and sharp IGC sites aligned in the L direction and about as deep as the white line representing the end of the $1.5 \mathrm{~mm}$ lip after $25.5 \mathrm{~h}$. The IGC density and length were approximately uniform along the full length of the sample.

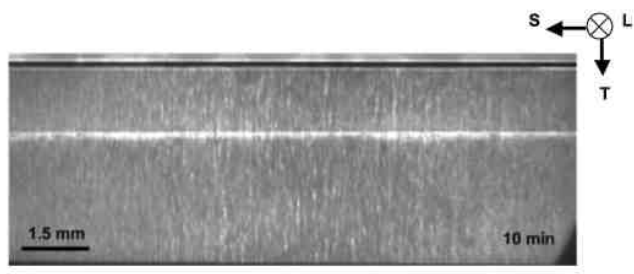

(a)

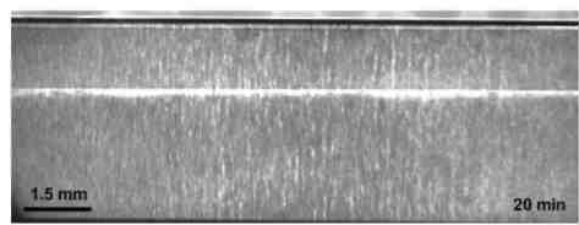

(b)

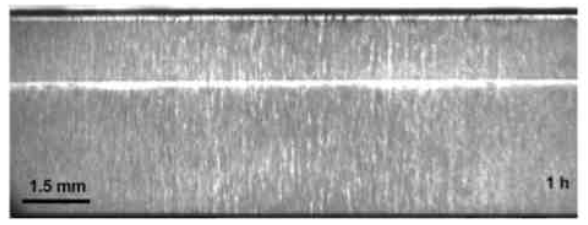

(c)

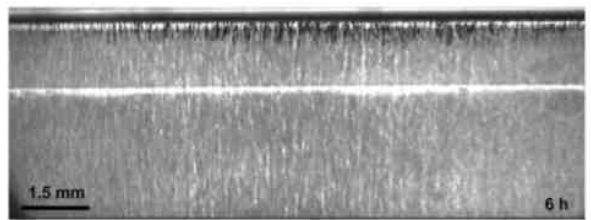

(d)

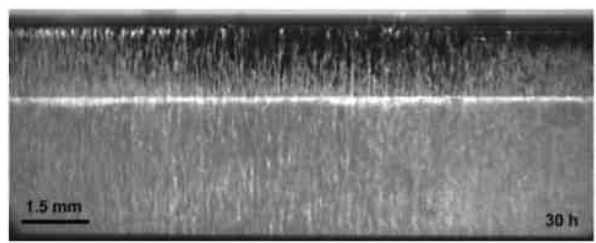

(e)

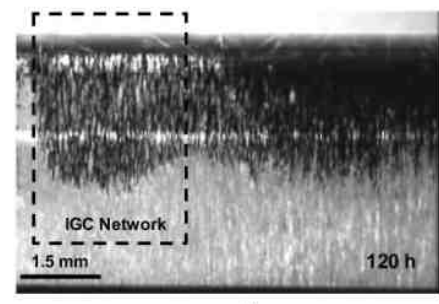

(f)

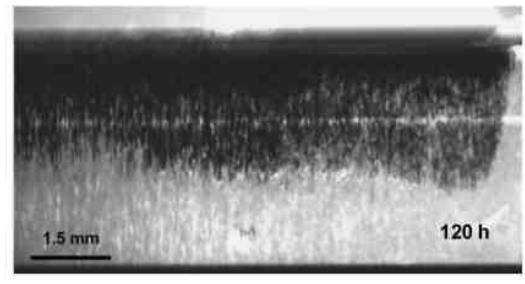

(g)

Figure 5. X-ray microfocal radiography images of IGC in unstressed T sample at $-580 \mathrm{mV}$ SCE in $1.0 \mathrm{M} \mathrm{NaCl}$ after (a) $10 \mathrm{~min}$, (b) $20 \mathrm{~min}$, (c) $1 \mathrm{~h}$, (d) $6 \mathrm{~h}$, (e) $30 \mathrm{~h}$, (f) $120 \mathrm{~h}$, left side of the sample, and (g) $120 \mathrm{~h}$, right side of the sample. 
Figure 5 shows a similar series of radiographic images for an unstressed T sample. These images exhibit different morphologies than those of the L sample in Fig. 4. The intermetallic particles are more distinct, reflecting the shorter grain dimensions in the T direction compared to the $\mathrm{L}$ direction. The images from the first $20 \mathrm{~min}$ (Fig. 5a and b) show almost no evidence of IGC, with attack just visible after $1 \mathrm{~h}$ of exposure (Fig. 5c). With longer exposure (Fig. $5 \mathrm{~d}$ and e), more IGC sites initiated and grew in the T direction but at a slower rate than in the $\mathrm{L}$ sample. Furthermore, the attack was not as straight and sharp as in the L sample. Figures $5 \mathrm{f}$ and g show higher magnification images on the left and right sides of the sample, respectively, after $120 \mathrm{~h}$ exposure. The intergranular corrosion is seen to link along the S direction, forming a network. Some white features, apparently unattacked grains, extruded out of the top surface, which might be attributed to stress generated by the corrosion products, as discussed below.

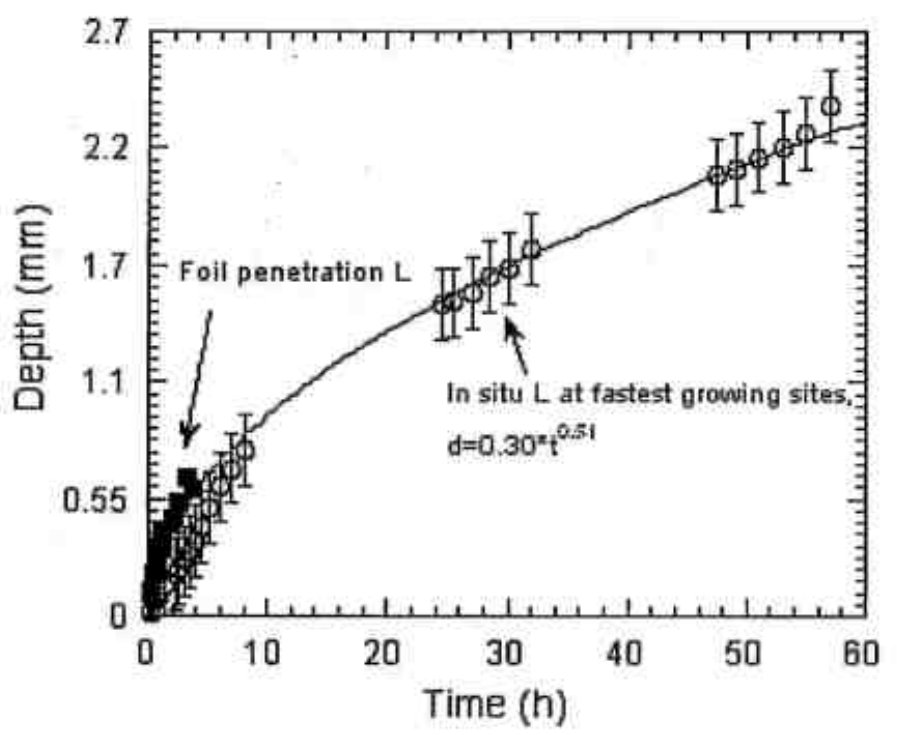

Figure 6. AA 2024-T3 at $-580 \mathrm{mV}$ SCE in $1.0 \mathrm{M} \mathrm{NaCl}$. Open symbols and line: fastest growing IGC sites for unstressed L samples from in situ radio-graphic experiments. The open symbols are average values of 20 sites and the bars represent the standard deviation. Filled symbols: foil penetration

data. 13

The lengths of the fastest growing cracks in $\mathrm{L}$ and $\mathrm{T}$ samples were measured as a function of time from the X-ray films and are shown in Fig. 6 and 7, respectively. Also shown in these figures are data from foil penetration experiments. ${ }^{12}$ Foil penetration experiments measure only the time for the first site to penetrate the foil, or the fastest growing site. ${ }^{15-19}$ The open circles in Fig. 6 represent the depth of the deepest site at any given time in the in situ radiographic experiment, which is a combination of the data from different sites. These data almost overlie the data from the foil penetration experiments under the same conditions. The similarity in the data from two different techniques suggests that both approaches provide accurate assessments of crack growth rates. However, the foil penetration technique does not provide information on the propagation rates of individual sites, only the nominal fastest rate for an ensemble of sites. ${ }^{11,18,19}$ These figures also show that the IGC kinetics measured over relatively short times using the foil penetration method are valid over the longer times of observation in the in situ radiography experiments. 


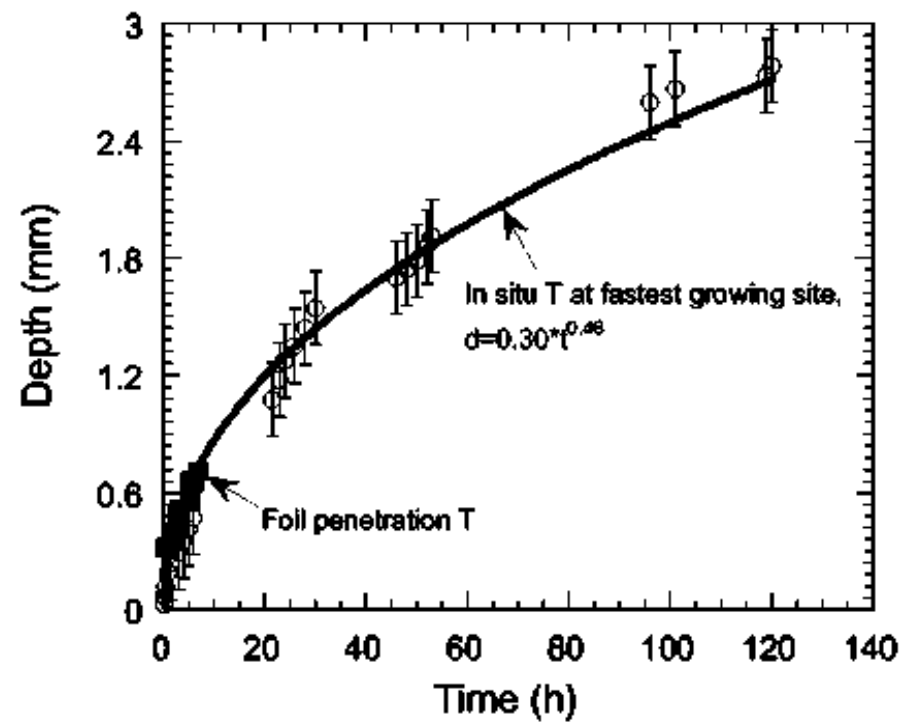

Figure 7. AA 2024-T3 at $-580 \mathrm{mV}$ SCE in $1.0 \mathrm{M} \mathrm{NaCl}$. Open symbols and line: fastest growing IGC sites for unstressed $\mathrm{T}$ samples from in situ radio-graphic experiments. The open symbols are average values of 20 sites and the bars represent the standard deviation. Filled symbols: foil penetration data. $^{13}$

In situ radiography of stressed $L-T$ sample.- IGC is constrained to the intergranular region, which is primarily between the grains elongated in the $\mathrm{L}$ and $\mathrm{T}$ directions of a wrought microstructure. In service, stress is commonly applied to such a microstructure along the $\mathrm{L}$ and $\mathrm{T}$ directions, or nominally parallel to the large grain boundaries. It is of interest to know how IGC, constrained by the microstructure to be parallel to an applied stress, develops into IG-SCC that is nominally perpendicular to the applied stress. To study this transition, an L-T sample was tested in the in situ radiographic experiment. The sample was stressed in the $\mathrm{T}$ direction with initial strain of $0.28 \%$ and the corrosion attack grew from the exposed L face in the L direction. X-rays penetrated through the sample thickness in the $\mathrm{S}$ direction. Figure 8 shows a time series of radiographic images of the L-T sample. These images exhibit quite different cracking morphology than those of the unstressed L sample in Fig. 4 and the unstressed T sample in Fig. 5. The IGC was wavy and more diffuse as it grew in the L direction. In the L-T orientation, the $\mathrm{X}$-rays illuminated the sample on the $\mathrm{S}$ face and propagated through the thickness of the sample in the $\mathrm{S}$ direction. It was previously reported that the average grain dimension in the $\mathrm{S}$ direction for the tested material at the $1 / 4 \mathrm{~T}$ section is approximately $50 \mu \mathrm{m} .{ }^{17,20}$ The thickness of the sheet sample used in the in situ X-ray radiography study was $1.05 \mathrm{~mm}$, indicating that there were about 21 grains through the thickness of this sample in the $\mathrm{S}$ direction. All of these grains were illuminated by the X-rays. Note that it is impossible to distinguish the individual IGC or intermetallic particles in this orientation. The radiographic image is an integration of all individual IG attack through the sample thickness in the S direction. The cracks were spread out on a plane that was perpendicular to the imaging beam, rather than perpendicular to the beam as in Fig. 4 and 5. The cracks appear wavy instead of straight as in the L sample because of the orientation of the IGC relative to the orientation of the X-ray imaging. Many cracks were found along the length of the sample, but one site became longer than the others and almost penetrated the sample after $78.5 \mathrm{~h}$ (Fig. 8d) In this image, the sample can be seen to be bent at the location 
of the long crack even though the ends were constrained by the uniaxial tensile stressing jig. This suggests that the corrosion products produced a wedging stress that deformed the sample. Figure 8e shows a sequence of images of the longest crack (indicated by an arrow in Fig. 8a) at higher magnification. Instead of growing directly downward in the L direction, the IGC spread along the grain boundaries in the $\mathrm{S}$ plane. Figure 9 is an unetched optical metallographic section of the $\mathrm{L}-\mathrm{T}$ sample after the experiment. The section is oriented in the same direction as the radiographic images. Most of the IGC was in another plane parallel to the section, and only the grain boundary regions in the $\mathrm{S}$ direction between the elongated grains are visible. Branching of the attack is seen at triple points where a grain boundary in the $\mathrm{L}$ direction ended.

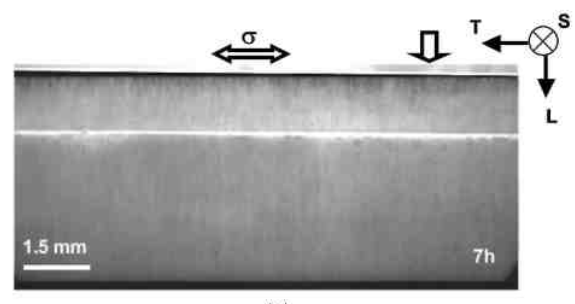

(a)

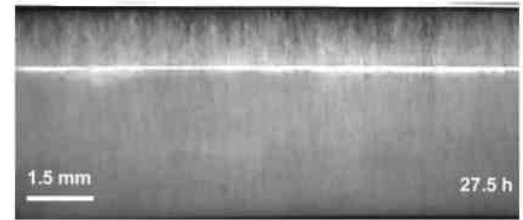

(b)

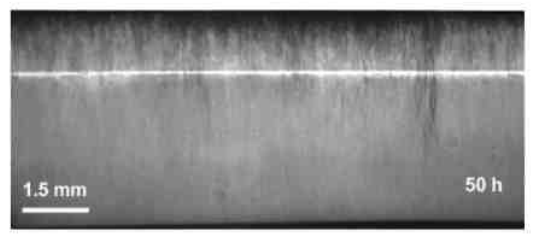

(c)

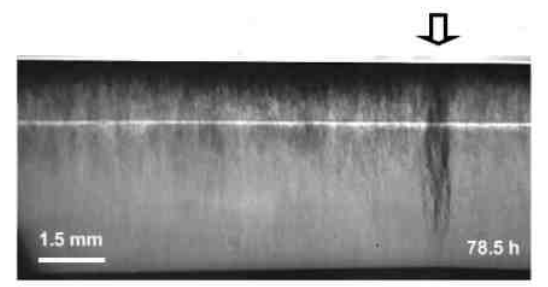

(d)

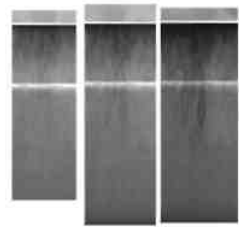

22.5h $\quad 30 \mathrm{~h} \quad 48 \mathrm{~h}$
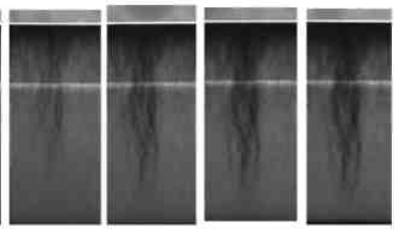

Figure 8. X-ray microfocal radiography images of IGSCC in L-T sample with initial $0.28 \%$ strain at $-580 \mathrm{mV}$ SCE in $1.0 \mathrm{M} \mathrm{NaCl}$ after (a) $7 \mathrm{~h}$, (b) $27.5 \mathrm{~h}$, (c) $50 \mathrm{~h}$, and (d) $78.5 \mathrm{~h}$, and (e) series of images of IGSCC at site by an arrow in Fig. 8a. 


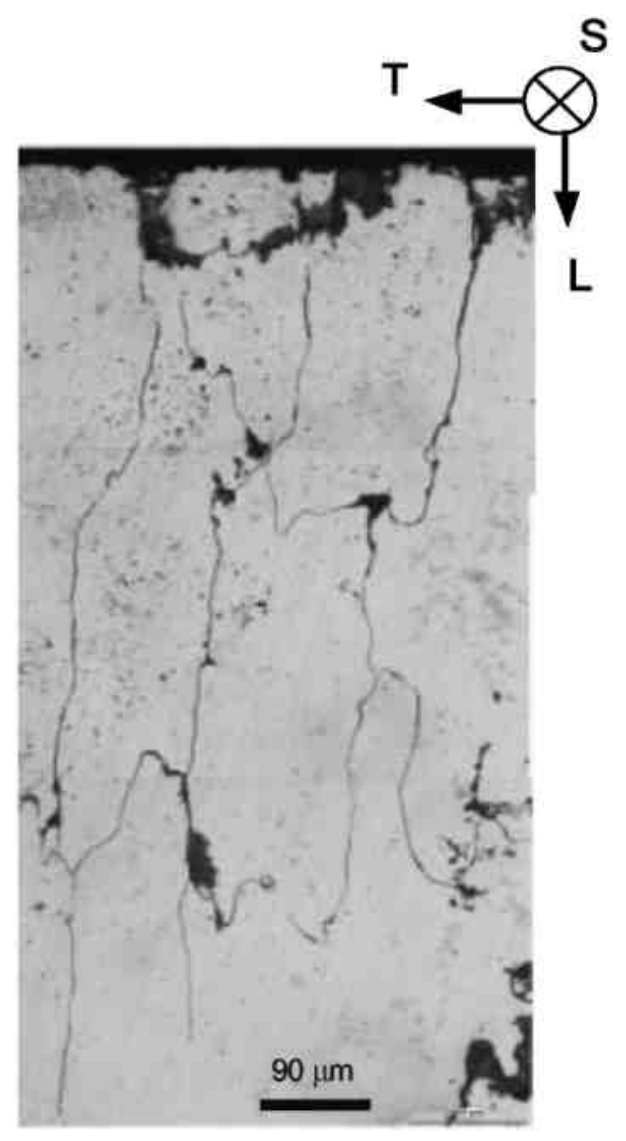

Figure 9. Metallurgical cross section of L-T sample after potentiostatic polarization at -580 mV SCE in 1.0 M NaCl.

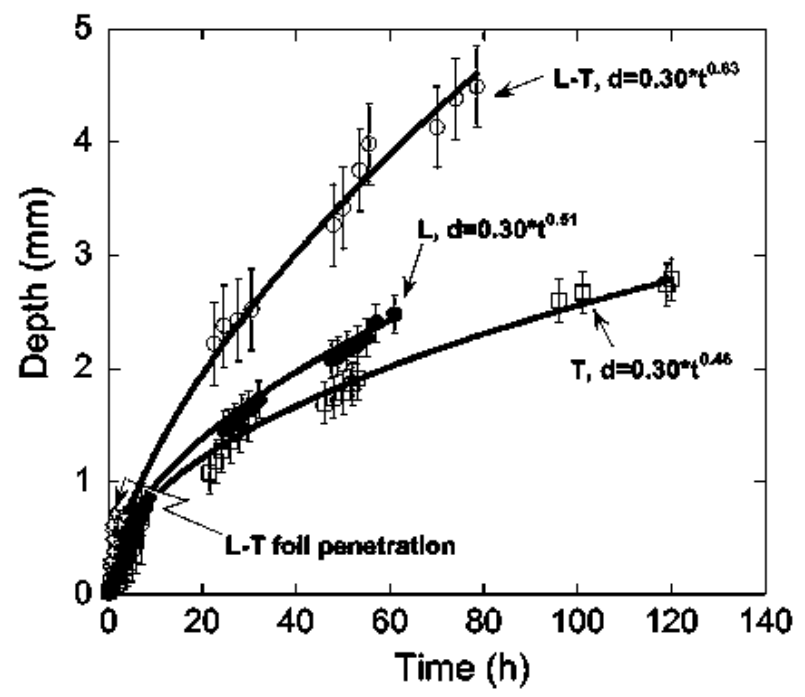

Figure 10. Growth rate of IGSCC for L, T, and L-T (0.28\%) samples at fastest growth sites at -580 mV SCE in 1.0 $\mathrm{M} \mathrm{NaCl}$ from in situ radiographic experiments. The symbols are average values of twenty sites and the bars represent the standard deviation. For comparison, L-T foil penetration data are shown as "+" symbols. 
Figure 10 shows the growth kinetics of IGC and IGSCC in the L-T sample, determined by measuring the depth of the deepest site as a function of time from the radiographic images shown in Fig. 8. Stressed L-T foil penetration data are also presented for comparison. ${ }^{12}$ As was the case for the unstressed samples, the results from the L-T foil penetration experiments overlie those from the L-T radiography experiments. Also shown in Fig. 10 are the IGC depths for unstressed $\mathrm{L}$ and $\mathrm{T}$ samples determined from radiographic experiments, shown in Fig. 4 and 5, respectively. Stress in the $\mathrm{T}$ direction has a small influence on the penetration rate relative to the unstressed condition. The L-T growth rate is much slower than was found previously for L-S and T-S samples, which were stressed in the through-thickness or S direction. ${ }^{11}$ The stressing direction and crack propagation orientation obviously have a large effect on the SCC growth kinetics. As a result of the Poisson effect, the application of a tensile stress in the $\mathrm{T}$ direction resulted in a compressive stress in the through-thickness direction, which is perpendicular to the IGC grain faces. Despite this compressive stress, the rate of penetration was increased by the applied tension in the T direction. This is discussed further below.

Ex situ radiography of stressed $L-T$ foil penetration sample. - Further clarification of the morphology of the L-T samples was obtained using a sample tested by the stressed foil penetration experiment. Details of this approach are given elsewhere, ${ }^{12}$ but in the foil penetration experiment, an area on the broad face of the sample is exposed to the solution rather than a thin edge, as in the in situ radiography experiment. Because of the configuration of the standard foil penetration experiment, it is not possible to obtain in situ radiographic images. Therefore, a foil penetration sample was examined by radiography after the experiment, when corrosion had just penetrated through the sample. Figure 11 shows radiographic and cross-sectional images for a foil penetration L-T sample. Similar to the L-T radiographic sample shown in Fig. 8, the sample was stressed in the $\mathrm{T}$ direction and the exposed face was an $\mathrm{L}$ plane, so the attack grew in the $\mathrm{L}$ direction. In this case, however, the X-rays penetrated through the sample thickness along the growth direction, or the $\mathrm{L}$ direction. The intermetallic particles are evident in this orientation (Fig. 11a). Also seen, and at higher magnification in Fig. 11b, are short dark lines elongated in the $\mathrm{T}$ direction. These are the IGC sites that penetrated the sample in the L direction. Figure 11c is an unetched metallographic section along the $\mathrm{T}$ direction, or loading direction. The cracks are seen to have grown in the L direction, which is perpendicular to the loading direction, but Fig. 11b shows that the primary crack face was in the S plane or parallel to the loading direction.

Fractography of unstressed and stressed L-T samples. - To better understand the transition between IGC and IGSCC, an unstressed but prenotched sheet L sample was potentistatically polarized in $1 \mathrm{M} \mathrm{NaCl}$ at $-580 \mathrm{mV}$ SCE for $4 \mathrm{~h}$. The sample had the same general shape and configuration as an L-T in situ sample $(80 \times 4 \times 2 \mathrm{~mm})$. Prior to the test, a notch $660-\mathrm{mm}$ deep in the $\mathrm{L}$ direction and $350-\mathrm{mm}$ wide in the $\mathrm{T}$ direction was cut using a diamond saw. The in situ cell was used to expose the thin edge containing the notch, which was an L surface, to solution, allowing IGC growth in the L direction. However, the sample was tested in the unstressed condition without the stressing jig. After the exposure, the sample was removed from the cell and pulled in tension in the T direction until it fractured, which occurred beneath the prenotch. Figure 12 shows the T-oriented fracture plane. All IG cracks propagated in the L direction from top to bottom. Regions ruptured by mechanical overload are denoted in this figure by the letter "R." These rupture regions separate parallel IGC regions, a large one of which is shown at the right part of Fig. 12. The individual IGC regions were not connected. Figures 4 and 11 also show that in the unstressed condition, IGC regions initiated and grew in L direction but were separated by neighboring grain bodies. 


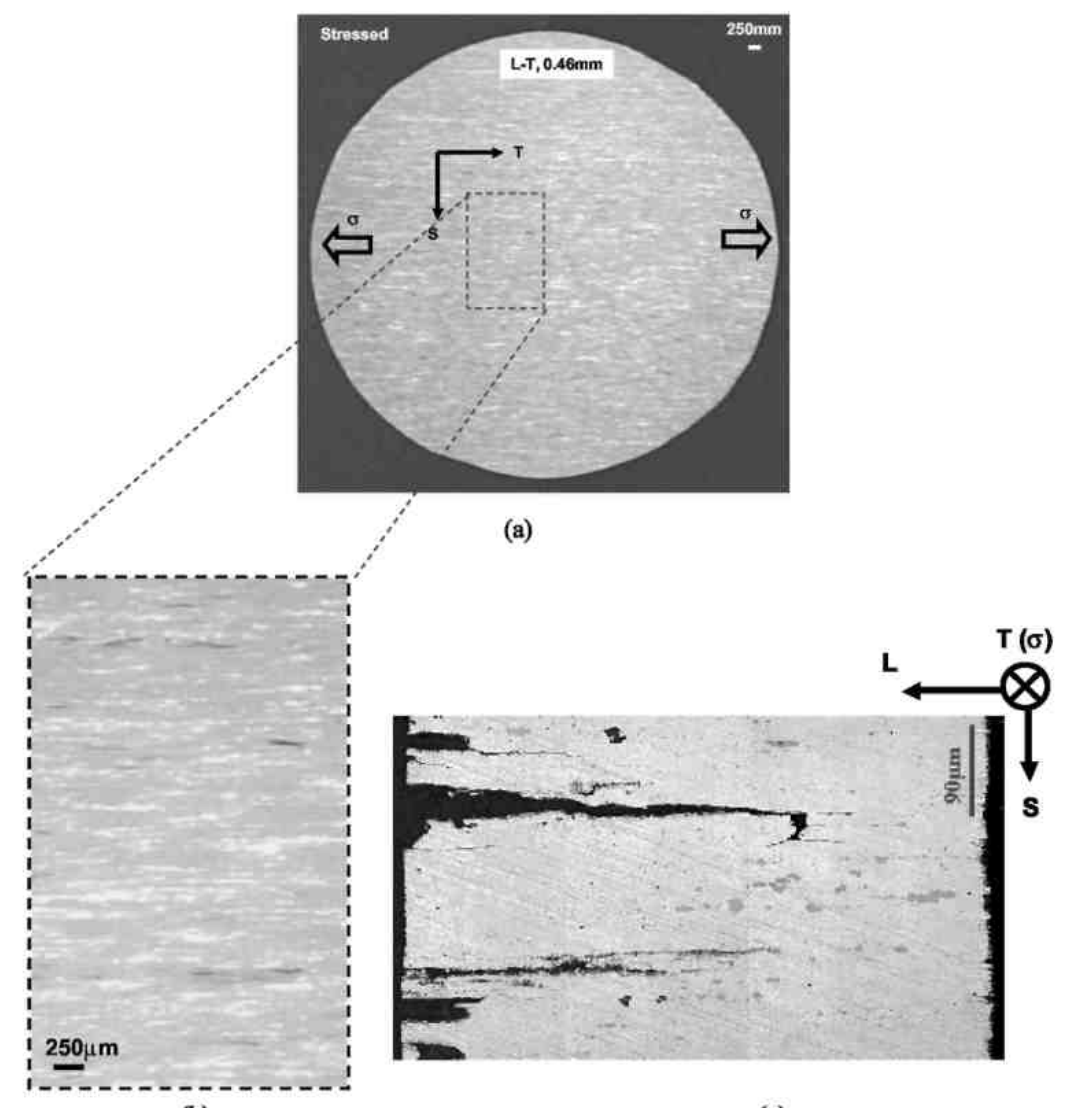

(b)

(c)

Figure 11. Images of L-T foil penetration sample after testing at $-580 \mathrm{mV}$ SCE in $1.0 \mathrm{M} \mathrm{NaCl}$ (a) radiographic image, (b) magnified radiographic image, and (c) optical cross section.

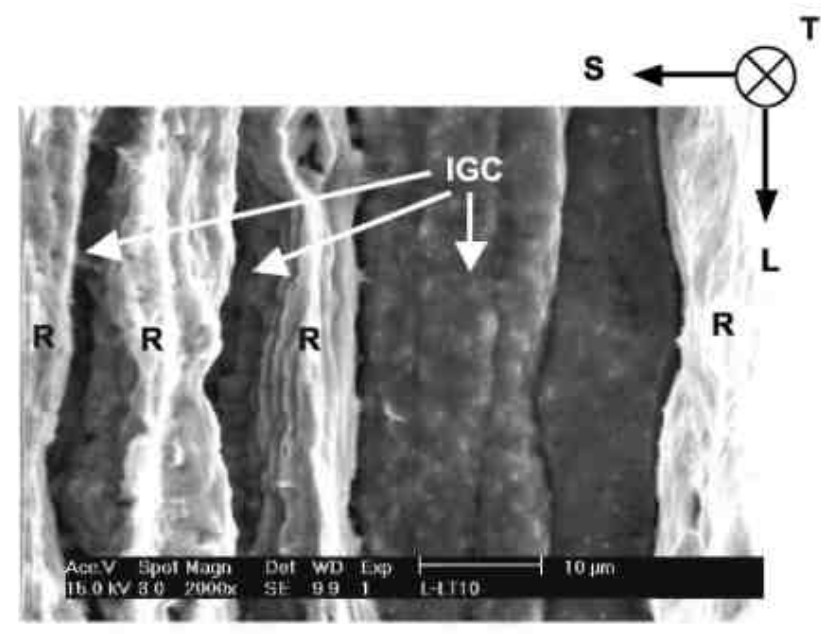

Figure 12. L-T sample after unstressed exposure at -580 $\mathrm{mV} \mathrm{SCE}$ in $1 \mathrm{M} \mathrm{NaCl}$ for $4 \mathrm{~h}$ and then mechanical overload fracture by stressing in T direction. Letter "R" designates the mechanical rupture region and arrows indicate IGC regions. 


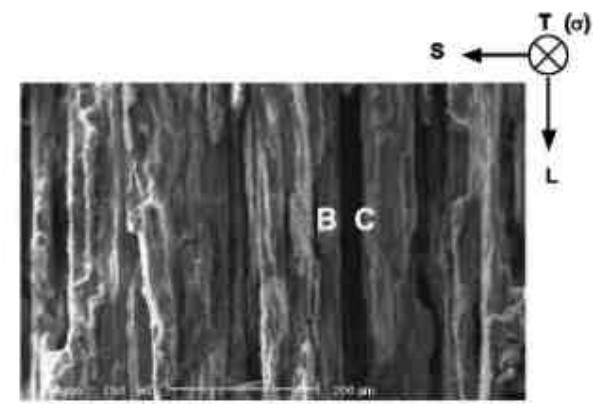

(a)

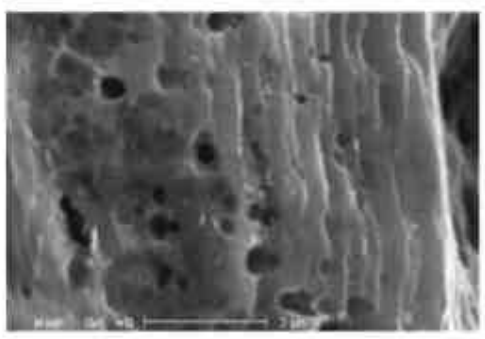

(b)

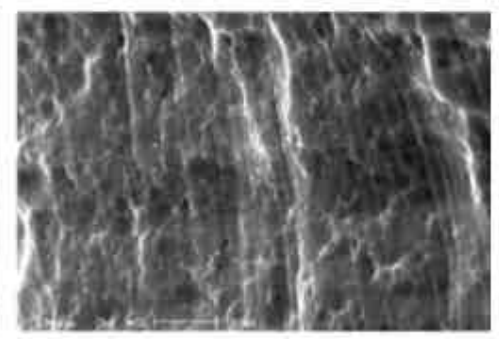

(c)

Figure 13. Secondary electron images of fractured L-T sample constrained in the stressing jig with fixed displacement (0.28\% initial strain): (a) low magnification of fracture surface, (b) high-magnification image of the region shown by the letter " $\mathrm{B}$ " in (a), and (c) high-magnification image of the region shown by the letter "C" in (a).

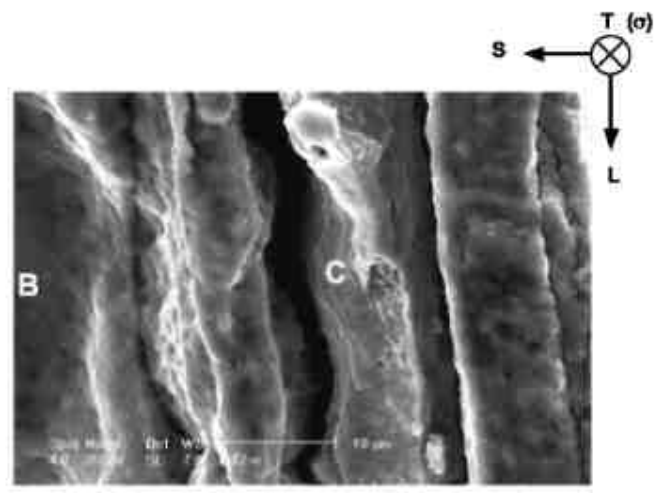

(a)

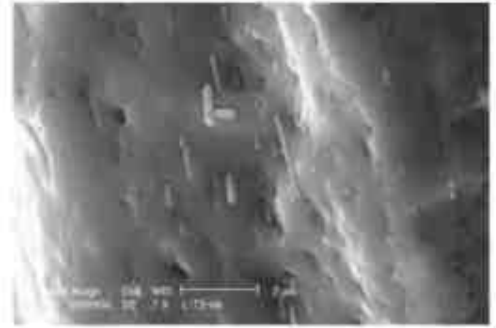

(b)

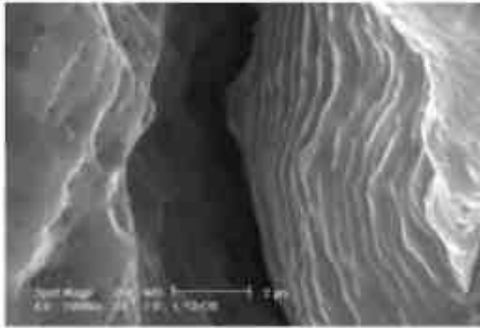

(c)

Figure 14. Secondary electron images for fractured L-T sample constrained in the stressing jig with fixed displacement (0.5\% initial strain): (a) low magnification of fracture surface, (b) high-magnification image of the region shown by the letter " $B$ " in (a), and (c) high-magnification image of the region shown by the letter "C" in (a). 
The fractography of stressed L-T samples is quite different. An L-T sample was given the same treatment as the one shown in Fig. 8; it was constrained in the stressing jig with fixed displacement $(0.28 \%$ initial strain) and polarized at $-580 \mathrm{mV}$ SCE in $1 \mathrm{M} \mathrm{NaCl}$ for $10 \mathrm{~h}$. The sample was pulled apart after the exposure, and the fracture surface is shown in Fig. 13. Again, the fracture surface had a nominal T orientation. Figure 13a exhibits IGSCC along the sides of the elongated pancake-shaped grains, with very little evidence of mechanical rupture. As mentioned previously, the average grain dimension in the $\mathrm{S}$ direction is about $50(\mu \mathrm{m}$. The topography in the $\mathrm{T}$ direction (normal to the page) is the result of a variation in the positions of the grain edges; some grains edges are recessed and some are raised due to this variation. Figures $13 \mathrm{~b}$ and c are high-magnification images of two relatively raised grain edges. They show lines aligned in the $\mathrm{L}$ direction that are apparent arrest marks. These lines are evidence of discontinuous sideways linking of the various IGC sites that grow in the L direction. Details of this linking process are described below, but it is the mechanism by which separated IGC sites growing in the L direction with S faces become IGSCC with a nominally T-oriented fracture surface. These high-magnification images also display many corroded holes and rod-shaped particles, which are associated with anodic dissolution on the grain facets, either during the IGC growth process or during subsequent exposure after the crack front passed by.

Another L-T sample was given the identical treatment as the sample in Fig. 13, except that the displacement was larger with an initial strain of $0.5 \%$. The fracture surface again shows a convoluted structure as a result of IGSCC at the grain edges, with no evidence of mechanical rupture (Fig. 14a). The higher magnification image in Fig. 14b shows a relatively smooth grain edge that is normal to the $\mathrm{T}$ direction. The rod-shaped particles are clearly seen as well as localized attack directly adjacent to them. However, compared with the L-T sample exposed at lower stress (0.28\% initial strain) shown in Fig. 13, this sample exhibited less localized attack, perhaps because of a greater rate of crack growth. The region shown at high magnification in Fig. 14c again exhibits arrest marks aligned in the L direction and indicating linkup of individual IGC sites.

These images suggest that there is a transition involving coalescence of individual IGC sites to IGSCC under applied elastic stress parallel to the primary IGC cracks. At the relatively high applied anodic potential (-580 mV SCE), IGC initiated and grew along the large plane grains. The transition to IGSCC was promoted by the applied stress in the transverse direction and the arrest marks indicate discontinuity in the transition process.

Current responses of $L$ and $L-T$ samples. - Figure 15a shows the current response from the L-T experiment shown in Fig. 8 and from an unstressed L experiment that was performed in the same configuration as the in situ radiography experiments (exactly like the experiment shown in Fig. 4, but a different run without radiographic imaging). Both samples were polarized at -580 $\mathrm{mV} \mathrm{SCE}$ in $1 \mathrm{M} \mathrm{NaCl}$. It is interesting that the current measured on the unstressed $\mathrm{L}$ sample was about twice that measured on the stressed L-T sample, even though the rate of corrosion growth was faster on the L-T sample, as shown in Fig. 15a. The explanation for this discrepancy lies in the number of corrosion sites in the two cases. Figures 4 and 8 show that the IGC density is much greater in the unstressed L sample than the crack density observed in the L-T sample. (Note that the orientation of the L sample was different than the L-T sample. The length of the unstressed $\mathrm{L}$ sample was the $\mathrm{S}$ direction rather than the T direction. Had stress been applied, it would have been an L-S sample. This difference in orientation, however, should not affect the current response.) The application of stress resulted in a focus of the attack at fewer sites relative 
to the unstressed case, which was found previously for S and S-T samples in foil penetration experiments. $^{12}$

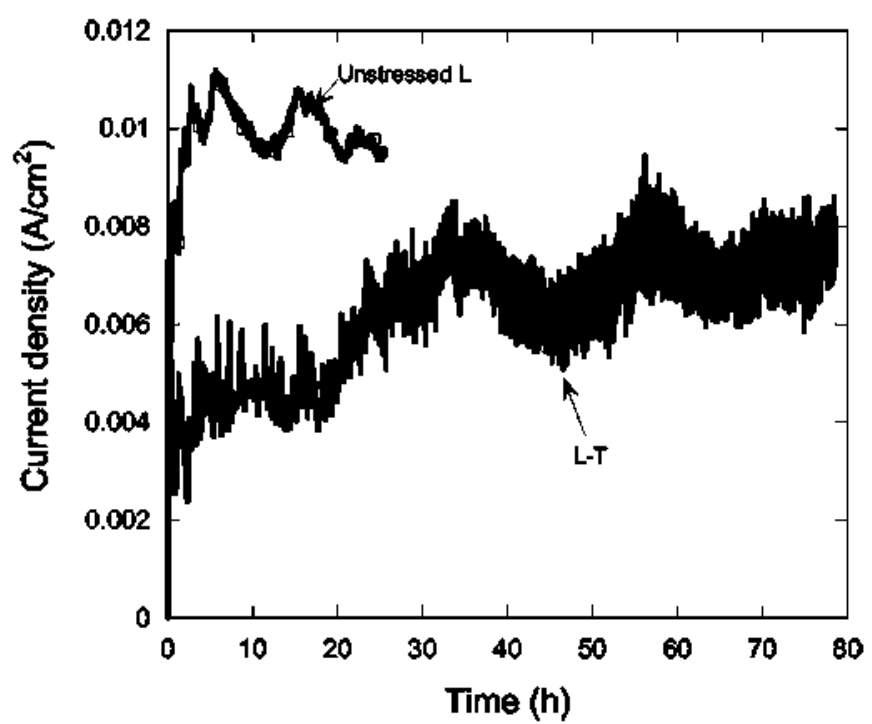

(a)

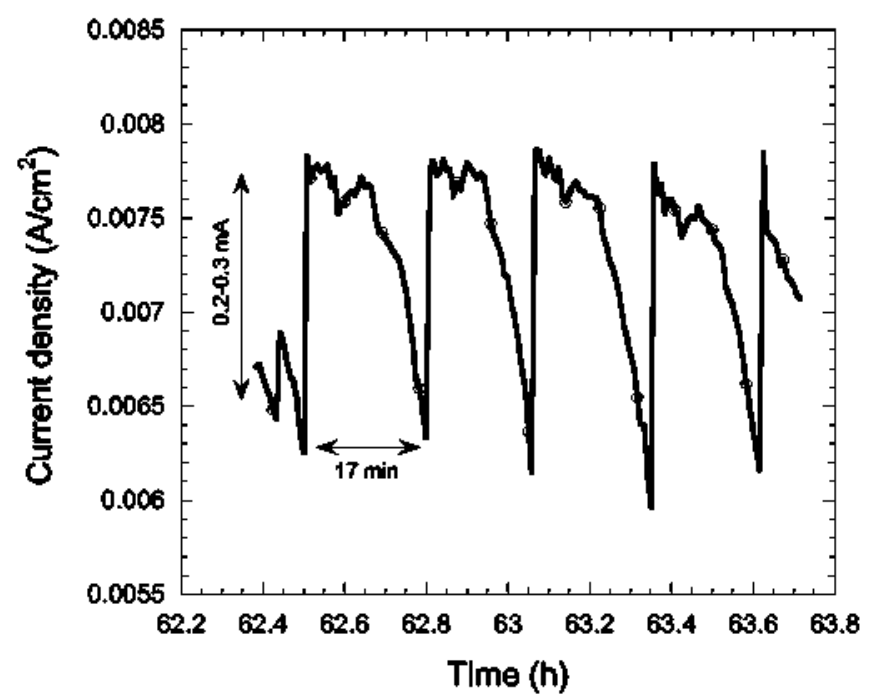

(b)

Figure 15. Current response during potentiostatic treatment at -580 mV SCE in $1 \mathrm{M} \mathrm{NaCl}$ : (a) comparison of current for unstressed L and the L-T sample, and (b) magnified view of oscillations in the current for the L-T sample.

The current for the L-T sample generally increased with time, but fluctuations at different frequencies are evident in Fig. 15a. A low-frequency oscillation with a period of about $30 \mathrm{~h}$ and amplitude of about $2.5 \mathrm{~mA} / \mathrm{cm}^{2}$ can be seen, as well as much higher frequency oscillations with lower amplitude. Figure 15b shows a magnified view of the current at the time of 62-64 h. Figure 8 shows that there was one dominant crack growing at this time, and the measured current might be primarily associated with the growth of that crack. During the time interval shown in Fig. 15b, there were several oscillations that were similar in form. The current increased rapidly, reached a 
plateau value, and then decayed to a low value before it spiked upward again.

Fast Fourier transformation (FFT) of the current was performed to analyze the current oscillations at different time periods. Figure 16 shows different 10 -h segments of the current response and the corresponding FFT spectra. During the first $10 \mathrm{~h}$ of the test, the current oscillations were relatively irregular (Fig. 16a). These fluctuations might be associated with the generation of new IGC sites as well as their propagation. The FFT in Fig. 16b shows that several frequencies were represented, but the major ones were $1.96 \times 10^{-4}$ and $3.55 \times 10^{-4} \mathrm{~Hz}$. At longer times, the oscillations became much more regular with only one dominant frequency. In the time period from 14 to $24 \mathrm{~h}$, the primary frequency observed, $3.86 \times 10^{-4} \mathrm{~Hz}$, was close to one of the major frequencies in the initial time period. As the time of the experiment increased, the major frequency increased to $9.0 \times 10^{-4} \mathrm{~Hz}$ and finally to $1.1 \times 10^{-3} \mathrm{~Hz}$. Both the current oscillations and the arrest marks on the fracture surfaces indicate that the crack growth was discontinuous on a scale that was not visible by the radiography. It is possible that the discontinuous growth was associated with the coalescence of the IGC into a crack perpendicular to the applied stress in the T direction.

\section{Discussion}

In this study, the transition from IGC to IGSCC was characterized for wrought AA2024T3 samples stressed in the T direction. In another report we present the results of stress applied in the $\mathrm{S}$ or through-thickness direction. ${ }^{11}$ However, the conditions of the current study are more relevant to service conditions, where stress is typically applied in an orientation along the elongated grains rather than perpendicular to them, e.g., an airplane fuselage. Because the applied stress was parallel to the elongated grains, the transition process is more complicated than for the case of stress applied in the through-thickness direction where the grain faces are normal to the stress. The acceleration of IGC/IGSCC growth rate by the application of tensile stress in the T direction was previously reported using the stressed oil penetration method. ${ }^{12}$ It is of interest to understand how this stress increases the growth rate despite the compression in the $\mathrm{S}$ direction induced by the Poisson effect and how IGC in the L direction along $\mathrm{S}$ faces becomes IGSCC with a fracture surface nominally perpendicular to the $\mathrm{T}$ applied stress.

IGC growth in the L direction for an unstressed sample proceeds rather straight down the large elongated grain boundaries that are perpendicular to the $\mathrm{S}$ direction in this wrought alloy. This is shown in the radiographic images in Fig. 4. IGC also occurs on some grain edges parallel to the $\mathrm{T}$ direction, as is evident in Fig. 12, which is the fracture surface for an unstressed sample that was pulled apart in the T direction after allowing extensive IGC attack. In that condition isolated grain edges were attacked, allowing easy separation at the grain boundary, while other grains were ruptured by the mechanical overload.

For a sample that is stressed in the $\mathrm{T}$ direction during exposure to conditions promoting IGC, the situation is different. Mechanical rupture of grain boundaries does not occur, but the IGC that is primarily on the large $\mathrm{S}$ faces and on some $\mathrm{T}$ grain edges must link up to generate a $\mathrm{T}$ fracture surface. The ex situ radiographic image of the L-T foil penetration sample in Fig. 11 shows that the attack primarily follows the large elongated grain boundaries that are perpendicular to the $S$ direction in this wrought alloy. The constraints of the foil penetration setup and samples prevent a clear view of these cracks, as they can only be observed ex situ and along the $\mathrm{L}$ growth direction. The in situ radiographic experiment setup allows for clear observation of the individual IGSCC sites, as in Fig. 8 for an L-T sample. 

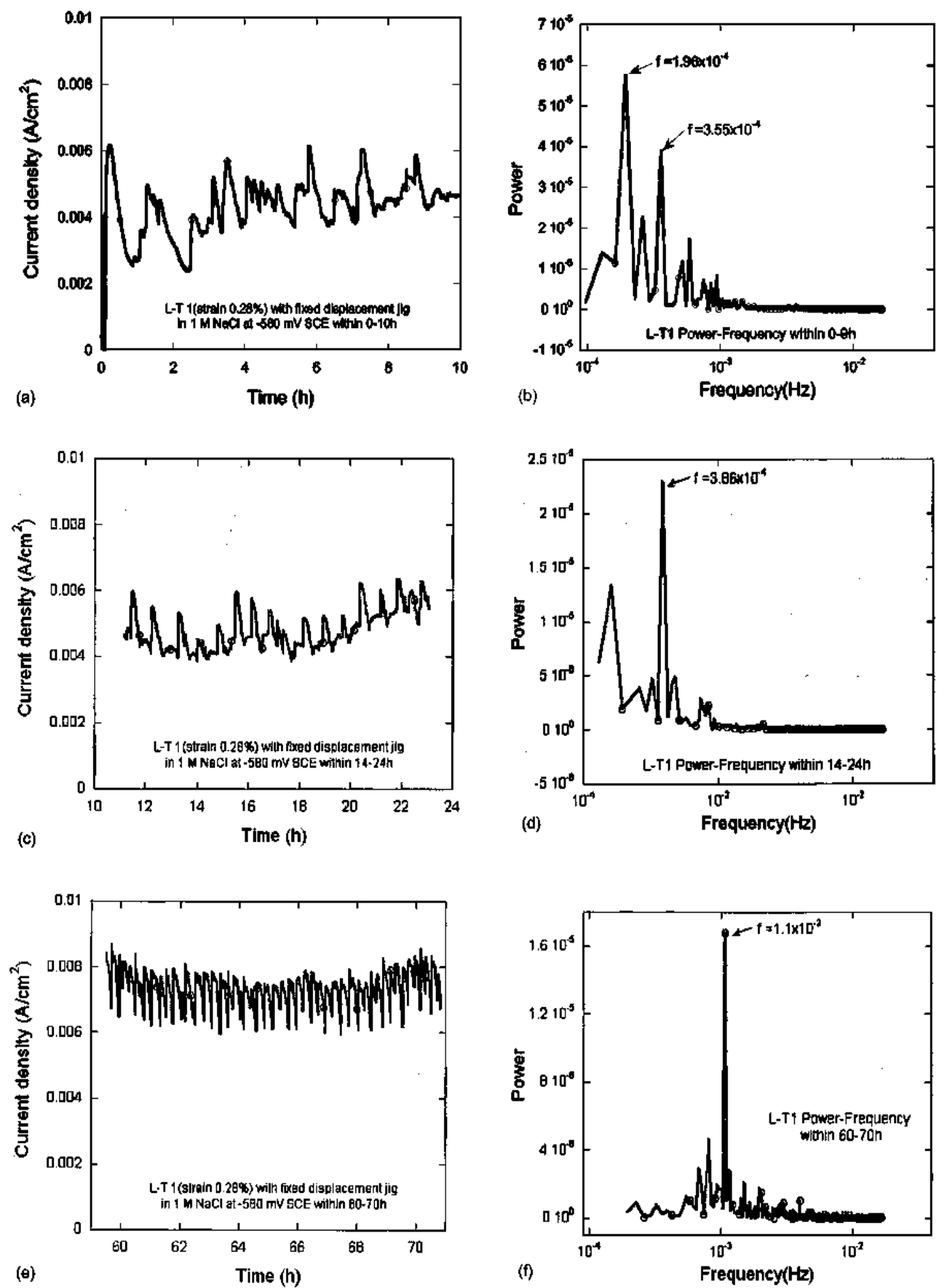

Figure 16. Partial current responses for different periods of time from the data for the L-T sample with $0.28 \%$ initial strain in Fig. 14 and their corresponding FFT spectra. (a-b) first 10h, (c-d) 14-24 h, (e-f) 60-70 h. 
In this orientation, the X-rays penetrate through the sample in the $\mathrm{S}$ direction or perpendicular to the primary plane of attack. The IGSCC appears to be wavy rather than straight because of the linking up of attack at various grains on various depths in the sample. The fracture surfaces of the stressed L-T samples shown in Fig. 13 and 14 provide more details about how the linking process proceeds. These images show a convoluted structure with $\mathrm{T}$ grain edges at different depths from the fracture surface due to the distribution of the grain edges through a sample. Some regions exhibit lines apparently associated with discontinuous attack. The lines are parallel to the nominal direction of penetration, the L direction, and suggest that the direction of discontinuous attack is in the $\mathrm{S}$ direction along the $\mathrm{T}$ edges of the elongated grains. So the linking up of IGC on the S faces occurs at the T edges after the IGC has passed to greater depths, and this linking is a discontinuous process. The linking of IGC along the grain edges results in transition to IGSCC and enhancement of growth kinetics. Comparing Fig. 13c and 14c, a higher initial strain, and thus stress, resulted in larger steps and thus a higher growth rate.

The current response generated by the potentiostatic treatment provides more evidence of the discontinuous nature of the attack. Figure 16 shows that for the sample with an initial strain of $0.28 \%$, current oscillations with a primary frequency of $1 \mathrm{mHz}$ are observed. Because the current response is for the whole exposed surface, which contains many linking sites, the frequency of events at each site would be much lower. Nonetheless, using $1 \mathrm{mHz}$ as an upper frequency limit and the $0.4-\left(\mu \mathrm{m}\right.$ spacing of arrest lines, an upper limit of $4 \times 10^{-7} \mathrm{~mm} / \mathrm{s}$ can be calculated for the discontinuous growth rate along the $\mathrm{S}$ direction, linking the IGC sites into IGSCC. This value is much less than the nominal growth rates in the $\mathrm{L}$ direction for the unstressed L or $0.28 \%$ strain L-T cases, $0.75-1 \times 10^{-5} \mathrm{~mm} / \mathrm{s}$. Even though the process is relatively slow, it is this linking of IGC sites that allows penetration in the L direction to occur at a higher rate than in the unstressed condition. This increase occurs despite the Poisson effect, which should close the IGC cracks and slow their growth. The primary effect of the T stress then is to provide a shear stress that drives the coalescence of the T edges of the IGC to form an IGSCC crack perpendicular to the applied stress in the $\mathrm{T}$ direction.

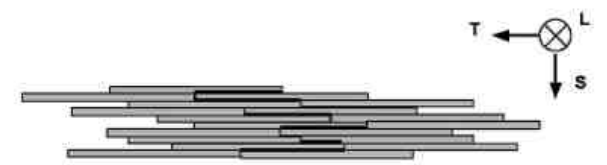

(a)

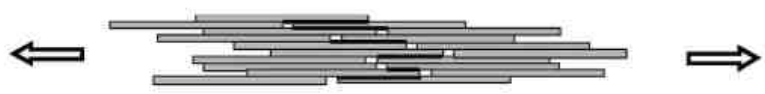

(b)

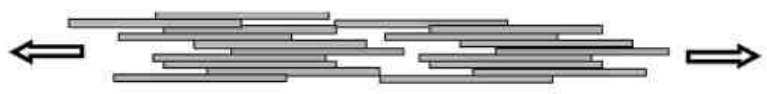

(c)

Figure 17. Schematic illustration for transition of IGC to IGSCC (viewed from L direction) in L-T sample. Rectangles represent the microstructure of an exposed $\mathrm{L}$ surface, with grains elongated in the $\mathrm{T}$ direction and thin in the S direction. (a) IGC prior to application of stress with dark lines indicating individual IGC sites, (b) initiation of IGC coalescence by application of stress in T direction, and (c) complete coalescence of IGC into IGSCC crack with nominal surface parallel to $\mathrm{T}$ direction. 
A schematic representation of the development of IGC propagating in the L direction into an IGSCC crack perpendicular to a stress applied in the $\mathrm{T}$ direction is shown in Fig. 17. The rectangles are meant to represent the microstructure of an exposed L surface, with grains elongated in the $\mathrm{T}$ direction and thin in the $\mathrm{S}$ direction. The grains are further elongated in the $\mathrm{L}$ direction perpendicular to the page. During the initial stages of exposure, (Fig. 17a), IGC is initiated and grows in the L direction along the grain boundaries. As the microstructure is weakened by this IGC growth, the grains start to slide apart along the $\mathrm{S}$ planes and open up along the grain edges on the T planes (Fig. 17b). This process is like a deck of cards being split apart. When the grain edges coalesce into a single crack (Fig. 17c), the transition from IGC to IGSCC is complete.

\section{Conclusions}

IGC and IGSCC of AA2024-T3 in the chloride solution were characterized using in situ and ex situ X-ray microradiography, SEM, and electrochemistry.

1. Applied tensile stress in the $\mathrm{T}$ direction results in a significant decrease in the breakdown potential and a small increase in the rate of IGC/IGSCC propagation in the L or rolling direction.

2. IGC occurs primarily along the large, flat faces of elongated grains. Tensile stress along the $\mathrm{T}$ direction promotes linking of the IGC on the edges of the elongated grains to form a fracture surface that is nominally perpendicular to the transverse stress.

3. Arrest marks on the fracture surface and current oscillations suggest that the linking process is discontinuous in nature and relatively slow compared to the rate of propagation in the L direction.

\section{Acknowledgments}

This work was supported by the United States Air Force Office of Scientific Research through grant no. F49620-02-1-0148 and FA9559-04-1-0368. The contract monitors were Lt. Col. Paul Trulove, Ph.D., and Major Jennifer Gresham, Ph.D.

The Ohio State University assisted in meeting the publication costs of this article.

\section{References}

1. M. O. Speidel, Metall. Trans. A, 6, 631 (1975).

2. D. O. Sprowls and R. H. Brown, in Proceedings of the Conference on Fundamental Aspects of Stress Corrosion Cracking, The Ohio State University, Columbus, OH (1967).

3. M. R. Bayoumi, Eng. Fract. Mech., 54, 879 (1996).

4. H. Vogt and M. O. Speidel, Corros. Sci., 40, 251 (1998).

5. C. S. Lee, Y. Choi, and I. G. Park, Metals and Materials International, 8(2), 191 (2002).

6. J. L. Searles, P. I. Couma, and R. G. Buchheit, Metall. Mater. Trans. A, 32A, 2859 (2001).

7. G. S. Frankel and Z. Xia, Corrosion (Houston), 55, 139 (1999).

8. J. E. Finnegan and W. H. Hart, in Stress Corrosion-New Approaches, H. L. Craig, Jr., Editor, American Society for Testing and Materials, West Conshohocken, PA (1976).

9. X. Zhao, G. S. Frankel, B. Zoofan, and S. I. Rokhlin, Corrosion (Houston), 59, 1012 (2003).

10. X. Liu, G. S. Frankel, B. Zoofan, and S. I. Rokhlin, in Symposium on Corrosion and Environmental Cracking of Aluminum, Corrosion in Marine and Saltwater Environments II, D. Shifler and T. Tsuru, Editors, PV 2005-14, p. 223, The Electrochemical Society Proceedings Series, Pennington, NJ (2004). 
11. X. Liu, G. S. Frankel, B. Zoofan, and S. I. Rokhlin, Corrosion (Houston), To be published (2005).

12. X. Liu, G. S. Frankel, B. Zoofan, and S. I. Rokhlin, Corros. Sci., 46, 405 (2004).

13. X. Liu, Ph.D. Thesis, The Ohio State University, Columbus, OH (2005).

14. B. Zoofan and S. I. Rokhlin, Mater. Eval., 52, 191 (1998).

15. A. Rota and H. Bohni, Werkst. Korros., 40, 219 (1989).

16. A. Rota and H. Bohni, Werkst. Korros., 40, 295 (1989).

17. W. Zhang and G. S. Frankel, Electrochem. Solid-State Lett., 3, 268 (2000).

18. F Hunkeler and H. Bohni, Corrosion (Houston), 37, 645 (1981).

19. F Hunkeler and H. Bohni, Corrosion (Houston), 40, 534 (1984).

20. W. Zhang and G. S. Frankel, J. Electrochem. Soc, 149, B510 (2002). 